\title{
The 0.4- $M_{\odot}$ eclipsing binary CU Cancri ${ }^{\star}$
}

\section{Absolute dimensions, comparison with evolutionary models and possible evidence for a circumstellar dust disk}

\begin{abstract}
I. Ribas ${ }^{\star \star}$
Departament d'Astronomia i Meteorologia, Av. Diagonal, 647, 08028 Barcelona, Spain

e-mail: iribas@am.ub.es

Received 23 July 2002 / Accepted 5 November 2002

Abstract. Photometric observations in the $R$ and $I$ bands of the detached M-type double-lined eclipsing binary CU Cnc have been acquired and analysed. The photometric elements obtained from the analysis of the light curves have been combined with an existing spectroscopic solution to yield high-precision (errors $\lesssim 2 \%$ ) absolute dimensions: $M_{\mathrm{A}}=0.4333 \pm 0.0017 M_{\odot}$, $M_{\mathrm{B}}=0.3980 \pm 0.0014 M_{\odot}, R_{\mathrm{A}}=0.4317 \pm 0.0052 R_{\odot}$, and $R_{\mathrm{B}}=0.3908 \pm 0.0094 R_{\odot}$. The mean effective temperature of the system has been estimated to be $T_{\text {eff }}=3140 \pm 150 \mathrm{~K}$ by comparing multi-band photometry (optical and infrared) with synthetic colors computed from state-of-the-art model atmospheres. Additionally, we have been able to obtain an estimate for the age $(\sim 320 \mathrm{Myr})$ and chemical composition $([\mathrm{Fe} / \mathrm{H}] \approx 0.0)$ of the binary system through its membership of the Castor moving group. With all these observational constraints, we have carried out a critical test of recent stellar models for low-mass stars. The comparison reveals that most evolutionary models underestimate the radius of the stars by as much as $10 \%$, thus confirming the trend observed by Torres \& Ribas (2002) for YY Gem and V818 Tau. In the mass-absolute magnitude diagram, CU Cnc is observed to be dimmer than other stars of the same mass and this makes the comparison with stellar models not so compelling. After ruling out a number of different scenarios, the apparent faintness of CU Cnc can be explained if its components are some $10 \%$ cooler than similar-mass stars or if there is some source of circumstellar dust absorption. The latter could be a tantalizing indirect evidence for a coplanar (Vega-like) dusty disk around this relatively young M-type binary.
\end{abstract}

Key words. binaries: eclipsing - binaries: spectroscopic - stars: low-mass, brown dwarfs - stars: fundamental parameters stars: individual: $\mathrm{CU} \mathrm{Cnc}$ - planetary systems: protoplanetary disks

\section{Introduction}

Low-mass stars constitute the most numerous stellar population of the Galaxy. Yet, their physical properties are still poorly known. This is not without the efforts of many investigators that have been working intensively on the modelling of the atmosphere and interior structure of sub-solar mass stars. The major problem that the field has to face is a conspicuous lack of suitable calibrators. Unfortunately, very few main-sequence M stars have empirically-determined masses, radii, luminosities and temperatures.

Eclipsing binaries have often served as valuable benchmarks for the validation of structure and evolution models (see, e.g., Andersen 1991). Despite some unsolved problems at the high-mass end of the main sequence, the behaviour of stars more massive than the Sun is well understood, partly thanks to the large number of eclipsing binaries with well-determined

\footnotetext{
* Tables 1 and 2 are only available in electronic form at the CDS via anonymous ftp to cdsarc.u-strasbg.fr (130.79.125.5) or via http://cdsweb.u-strasbg.fr/cgi-bin/qcat?J/A+A/398/239 $\star \star$ e-mail: iribas@am.ub.es
}

physical properties. The situation is radically different at the low-mass end of the main sequence. Selection effects caused by the faintness of the stars and the often strong intrinsic variations due to magnetic activity pose a serious challenge for the discovery and analysis of detached eclipsing binaries. Only three detached eclipsing systems with dwarf M-type components have been identified to date ${ }^{1}$. The member of the Castor multiple system YY Gem has almost identical components of spectral type M1 Ve and mass $\sim 0.6 M_{\odot}$ (Torres \& Ribas 2002, hereafter TR02). CM Dra holds the record for the lowest mass eclipsing binary known and has components of $\sim 0.25 M_{\odot}$ and spectral type M4.5 Ve (Metcalfe et al. 1996). These were the only two detached M-type eclipsing binaries known for decades until Delfosse et al. (1999a, hereafter D99) recently reported the discovery of eclipses in CU Cnc.

\footnotetext{
${ }^{1}$ Note that another dwarf M-type eclipsing binary was reported and analysed by Maceroni \& Rucinski (1997). The system, which was discovered by the OGLE survey, is very faint $(V \approx 18.3)$ and the physical properties of the components are currently not well constrained. Moreover, the light curve solution indicates strongly distorted components with the primary possibly filling its Roche lobe.
} 
CU Cnc (GJ 2069A, HIP 41824, $\alpha=8^{\mathrm{h}} 31^{\mathrm{m}} 37 \mathrm{~s} .58$, $\delta=+19^{\circ} 23^{\prime} 39^{\prime}$.5) is an 11.9-mag spectroscopic binary with M3.5 Ve components. Also, it has a fainter visual companion, CV Cnc or GJ 2069B, at an angular distance of $\sim 12^{\prime \prime}$. GJ 2069B is itself a close binary star (Delfosse et al. 1999b) with a mean spectral type of M4 and appears to form a physically bound quadruple system with CU Cnc. Interestingly, the masses found by D99 place CU Cnc nicely in between of YY Gem and CM Dra and make it a system of paramount importance to improve upon our knowledge of the physics of lowmass stars.

Currently, the high-precision spectroscopic orbit of CU Cnc presented by D99 (errors in minimum mass below 0.4\%) is not matched by the photometric light curves, and the authors reported only sparse photoelectric observations. The motivation of our study was to acquire and analyse new high-quality photometric observations with the aim of determining accurate masses and radii for the components of CU Cnc. With a careful estimation of the temperature, our goal is to compare the physical properties of CU Cnc's components with the predictions of the available low-mass stellar evolution models.

\section{Light curve observations}

The just recent discovery of the eclipsing nature of $\mathrm{CU} \mathrm{Cnc}$ is likely to be a consequence of its faintness but also, very importantly, of its shallow and short eclipses. The photometric observations published by D99 indicate a system with an orbital period of 2.77 days, eclipses of only $\sim 0.2$ mag in depth and about 2 hours in duration. However, the high scatter of these light curves and their poor phase coverage prevented an accurate determination of the physical properties of $\mathrm{CU}$ Cnc.

We report here new photometry of CU Cnc in the Johnson $R$ and $I$ bands. Both the photometric accuracy (a few millimagnitudes) and the phase coverage (over 2100 observations) are sufficient to guarantee a reliable determination of the light curve parameters thus permitting a critical evaluation of stellar models. The observations were carried out with the Four College $0.8-\mathrm{m} \mathrm{APT}$, which is equipped with a refrigerated Hamamutsu photoelectric detector and filters closely matching the standard Johnson system. Differential photometry was obtained in which HD 72093 (F8, $V=7.80$ ) was employed as the comparison star, while HD 72358 (F5, $V=8.31$ ) served as the check star. Although it is common practice to employ comparison stars that match the spectral type of the variable star, we preferred to avoid G, K or M stars because of concerns with magnetic-related intrinsic variability. In contrast, late $\mathrm{F}$ stars are known to be photometrically very stable. Note that only precise $R$ and $I$ photometry could be obtained with our instrumental setup because of the faintness of CU Cnc at shorter wavelengths. The system is so red that at the $I$ band, its brightness rises up to about $I \approx 9$ mag. No evidence of significant light variations (down to the few milimagnitude level) was found for the comparison-check star sets. The observations were reduced using photometric reduction programs at Villanova University (USA). Differential extinction corrections were applied, although these corrections were typically very small.
There is one feature that makes our photometric dataset especially noteworthy: the $\sim 2100$ measurements were obtained over a period of only 110 days (from December 5, 1998 through March 25, 1999). Classified as a flare star, CU Cnc is expected to be magnetically active and display some level of brightness variability due to surface inhomogeneities. Thus, the photometric observations were acquired over a short time span to minimise possible variations in the out-of-eclipse shape caused by starspot migration. Also, special care was taken to schedule the observations near the eclipses so that a very dense phase coverage could be achieved. To compute orbital phases, we adopted the ephemeris of D99 but correcting the spectroscopic reference epoch to the primary eclipse:

$T($ Min I $)=$ HJD $2450208.5068+2.771468 E$.

The phased light curves are presented in Fig. 1. Note the dense phase coverage, the relative shallowness of the eclipses and the out-of-eclipse variations presumably due to starspots. The individual photometric observations in the $R(n=2157)$ and $I$ $(n=2188)$ bands are provided in Tables 1 and 2 (HJD, phase and $\Delta \mathrm{mag}$ ), respectively, which are only available in electronic form.

As mentioned above, $\mathrm{CU}$ Cnc has an optical companion at $\sim 12^{\prime \prime}$. The diaphragm used for the photometric observations was $45^{\prime \prime}$ in diameter, so the light of the companion was included in the measurements. To estimate exactly the fraction of contributed light we made use of CCD images taken with the ALFOSC instrument at the Nordic Optical Telescope (La Palma). Several images in the $V$ and $I$ passbands were kindly acquired by R. J. Irgens on May 10, 1999 (HJD 2451309.375) at an orbital phase of 0.215. The reduction was carried out in the classical manner and aperture photometry was performed to estimate the differential magnitude between $\mathrm{CU} \mathrm{Cnc}$ and its companion. We obtained values of $\Delta V=1.45 \pm 0.01$ and $\Delta I=1.20 \pm 0.01$, which translates into the following fractions of $3 \mathrm{rd}$ light, that can be directly incorporated into the analysis of the light curves:

$F_{3}^{V}=0.21 \pm 0.01 ; \quad F_{3}^{I}=0.25 \pm 0.01$.

From these we estimate the fraction of 3rd light in the $R$ passband to be $F_{3}^{R}=0.23 \pm 0.01$.

\section{Light curve analysis}

The fits to the light curves were performed using an improved version of the Wilson-Devinney program (Wilson \& Devinney 1971; hereafter W-D) that includes a model atmosphere routine developed by Milone et al. (1992) for the computation of the stellar radiative parameters. A detached configuration was chosen for all solutions. Both reflection and proximity effects were taken into account, even though the light curves do not show strong evidence for these. The bolometric albedo was set at the canonical value of 0.5 for stars with convective envelopes. The gravity-brightening coefficient was adopted as 0.2 following the theoretical results of Claret (2000a). A mass ratio of $q=M_{\mathrm{B}} / M_{\mathrm{A}}=0.9184$ was adopted from D99 and the temperature of the primary component (eclipsed at phase 0.0 ) was 


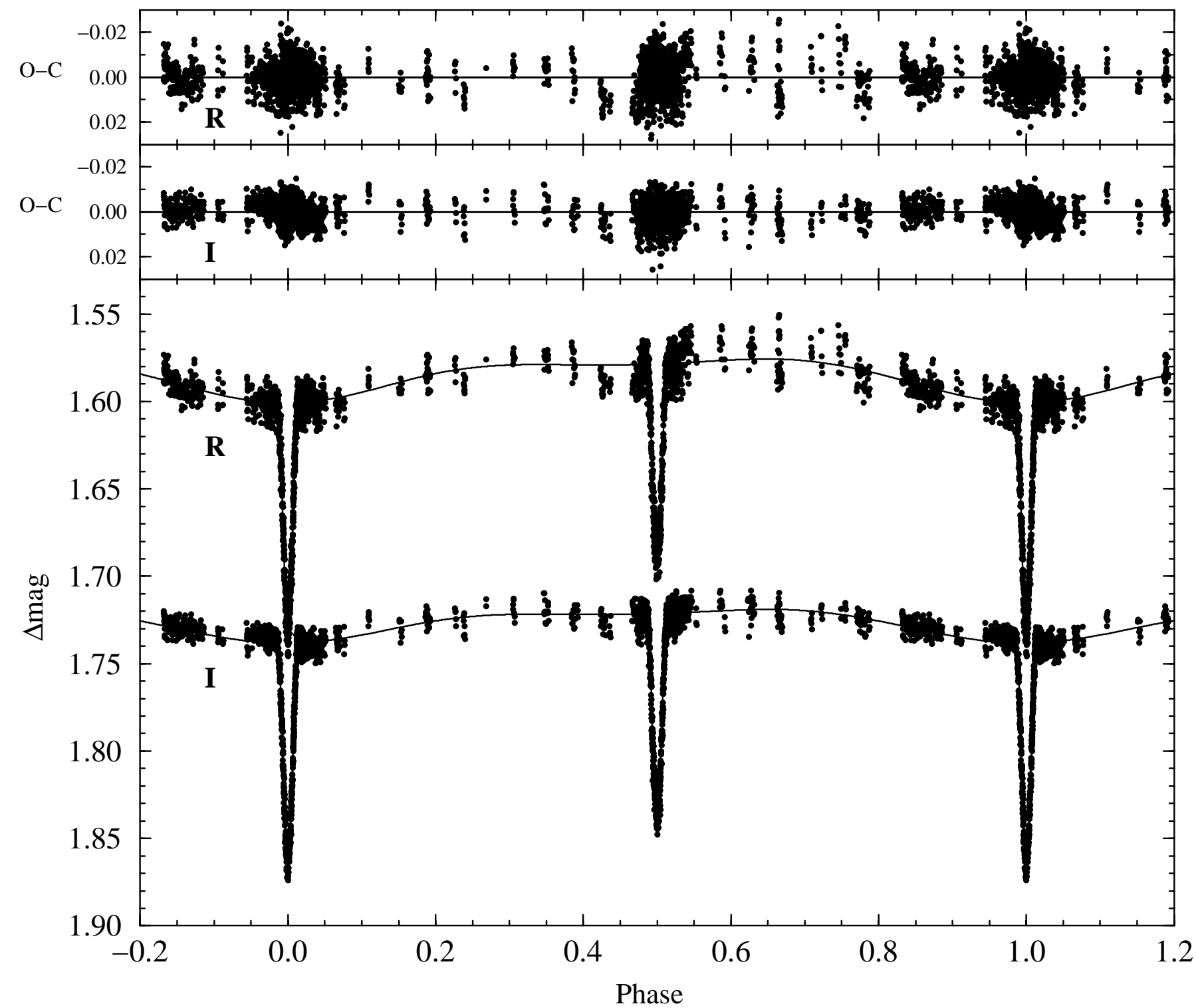

Fig. 1. $R$ and $I$ light curves of CU Cnc. A zero point shift of -1.45 has been applied to the $R$ photometry for display purposes. The solid line in the bottom panel is the best-fitting synthetic light curve generated by W-D. The top panels show the residuals of the fits. Enlargements of the fits to the eclipse phases are shown in Fig. 3.

set to $3160 \mathrm{~K}$ (see Sect. 4 for discussion). For the limb darkening we used a logarithmic law as defined in Klinglesmith \& Sobieski (1970). In our W-D implementation, first- and secondorder limb darkening coefficients are interpolated at each iteration from a set of tables computed in advance using a grid of Kurucz model atmospheres. However, CU Cnc has a temperature somewhat below the coolest model and a extrapolation had to be made. We also ran some tests by fixing the coefficients at the values computed by Claret (2000b) for the appropriate effective temperatures. The change had negligible effects but we finally adopted this latter prescription to avoid extrapolations. Finally, third light fractions were set to the values provided in Sect. 2.

The iterations with the W-D code were carried out automatically until convergence, and a solution was defined as the set of parameters for which the differential corrections suggested by the program were smaller than the internal probable errors on three consecutive iterations. As a general rule, several runs with different starting parameters are used to make realistic estimates of the uncertainties and to test the uniqueness of the solution.

Simultaneous W-D fits to the $R I$ light curves were carried out using the mean error of a single measurement as the relative weight, $w_{\lambda}$, of each passband ("curve-dependent" weighting scheme). Note that the number of photometric measurements we have is so large that 4-point normals had to be computed at the densest phases in or around the eclipses when running the W-D fits. We initially solved for the following light curve parameters: the orbital inclination $(i)$, the temperature of the secondary $\left(T_{\text {eff }}\right)$, the gravitational potentials $\left(\Omega_{\mathrm{A}}\right.$ and $\left.\Omega_{\mathrm{B}}\right)$, the luminosity of the primary at each passband $\left(L_{\mathrm{A}}\right)$, a phase offset $(\Delta \phi)$, and the spot parameters. The orbital eccentricity 
was set to zero as the light curves do not show any evidence for eclipses of different width or a secondary eclipse at an orbital phase other than 0.5 . Some tests were run to check for the possibility of a small non-zero orbital eccentricity. The W-D solutions converged towards a value of $e=0.001 \pm 0.003$, thus consistent with our adoption of a null eccentricity.

As expected for a system with partial eclipses and similar components, numerous test solutions revealed that the ratio of the radii $\left(k \equiv r_{\mathrm{B}} / r_{\mathrm{A}}\right.$, where $r_{\mathrm{A}}$ and $r_{\mathrm{B}}$ are the fractional radii in units of the separation) is poorly constrained. The problem is, in principle, severe because it implies that the light curves are not sufficiently sensitive to discriminate between the sizes of the components ${ }^{2}$. Thus, some source of external information has to be used to infer a value for $k$. With the ratio of temperatures well determined from the light curves, a spectroscopic estimation of the luminosity ratio would provide the necessary information.

Two high resolution and $S / N$ echelle spectra were obtained with the UES instrument at the William Herschel Telescope (La Palma) as part of the Service Programme. The observations were carried on March 8-9, 2001 (HJD 2451977.499) at orbital phase 0.287 . The raw images were reduced using standard NOAO/IRAF tasks (including bias subtraction, flat field correction, sky-background subtraction, cosmic ray removal, extraction of the orders, dispersion correction, merging, and continuum normalization). Equivalent width ratios for the components were measured for 10 relatively clean and isolated $\mathrm{Ca}$ I and $\mathrm{Fe}$ I absorption features. The average of the individual values yielded $E W_{\mathrm{B}} / E W_{\mathrm{A}}=0.73 \pm 0.05$ at a mean wavelength of $\lambda=5900 \AA$. Since the effective temperatures of both components are very similar, as shown below, the equivalent width ratio is analogous to luminosity ratio. From this we derive values of $\left[L_{\mathrm{B}} / L_{\mathrm{A}}\right]_{V}=0.72 \pm 0.05,\left[L_{\mathrm{B}} / L_{\mathrm{A}}\right]_{R}=0.74 \pm 0.05$ and $\left[L_{\mathrm{B}} / L_{\mathrm{A}}\right]_{I}=0.76 \pm 0.05$, for the luminosity ratios at the $V, R$ and $I$ passbands, respectively. Our value for the $V$-band luminosity ratio is indeed in good agreement with that obtained by D99. Although not explicitly given in the paper, Delfosse (2000, priv. comm.) kindly computed the luminosity ratio from their spectra and obtained a value of 0.68 , which lies within one sigma of our determination.

In practice, the luminosity ratio or the ratio of radii is not one of the parameters considered explicitly in the W-D code. Instead, are the surface potentials of the stars (which, along with $q$, control the sizes) that can be fixed or left free. We thus ran a number of solutions by adopting different values for the potential of the primary star until reaching agreement between the resulting luminosity ratio and the observed one. This occurred for $\Omega_{\mathrm{A}}=19.0$. All further solutions were ran by fixing $\Omega_{\mathrm{A}}$ at this value.

The shape of the out-of-eclipse region in the light curve clearly indicates that one or both components have surface inhomogeneities. A feature incorporated by the W-D program is the capability of fitting a simple spot model. The spots, assumed to be circular in shape, are described by W-D through

\footnotetext{
${ }^{2}$ Note, however, that the sum of fractional radii is extremely well determined because it depends strongly on the total duration of the eclipses.
}

four parameters, namely, the longitude, latitude, angular radius, and temperature ratio (spot relative to photosphere). Some of the spot parameters must be fixed when running solutions to prevent lack of convergence. For example, the latitude of the spots can be inferred theoretically. Granzer et al. (2000) find that a star such as the components of $\mathrm{CU}$ Cnc $\left(0.4-M_{\odot}\right.$ star in the ZAMS with an angular velocity 10 times the solar value) favours strongly the formation of spots at latitudes of about $60^{\circ}$. Tests ran with W-D indeed indicated a marginally better fit for spots at high latitudes.

As it seems obvious, the radius of the spot and the temperature contrast are strongly correlated. No empirical information is available on the temperature ratio between the spots and the surrounding photosphere for CU Cnc. However, Hatzes (1995) analysed Doppler tomography of the M-type eclipsing binary YY Gem and found the presence of dark areas cooler than the photosphere with $T_{\text {spot }} / T_{\text {phot }}=0.87-0.92$. For CU Cnc, we expect similar values. We thus ran a series of solutions and tested a variety of spot combinations (number of spots, locations, sizes, temperature ratios, etc.) to achieve the best possible fit to the light curves (see a detailed discussion of a similar procedure in TR02). Our preferred solution calls for two spots - one on each component - The spot on the primary component, which transits the central meridian at orbital phase 0.438 , appears to be relatively small, with a radius of $9^{\circ}$, and $\sim 450 \mathrm{~K}$ cooler than the photosphere. In contrast, the secondary component has a much larger spot with a radius of $31^{\circ}$ and a temperature difference with the surrounding photosphere of $\sim 200 \mathrm{~K}$. The center of the dark area transits the central meridian at orbital phase 0.997 . Its large size and relatively small temperature difference seems to indicate that this is more likely a spot complex rather than a homogeneous spot. Thus, the parameters determined represent an average over an extended photospheric area probably covered with patchy dark spots.

The photometric effect of the spot configuration adopted here is illustrated in Fig. 2. Note that the spot solution has only a mild effect on the rest of the light curve parameters. Furthermore, the spot solution is not unique and other configurations may be equally valid from a strictly numerical point of view.

The best-fitting two-spot model described above yields rms residuals of $0.008 \mathrm{mag}$ and $0.005 \mathrm{mag}$ for the $R$ and $I$ light curves, respectively. These small values reflect both the quality of the photometry and the excellent performance of the spot model. The synthetic light curve superimposed to the observations can be seen in Fig. 1, together with the residuals of the fit for each light curve. Also, for illustrative purposes, we show a detail of the eclipse phases in Fig. 3. The solution indicates that the eclipses are partial and that $\sim 25 \%$ of each component's flux is blocked during the corresponding eclipses. The parameters resulting from the light curve fit are listed in Table 3. The uncertainties given in this table were not adopted from the formal probable errors provided by the W-D code, but instead from numerical simulations and other considerations. Several sets of starting parameters were tried in order to explore the full extent of the parameter space. In addition, the W-D iterations were not stopped after a solution was found, instead, the program was kept running to test the stability of the solution 


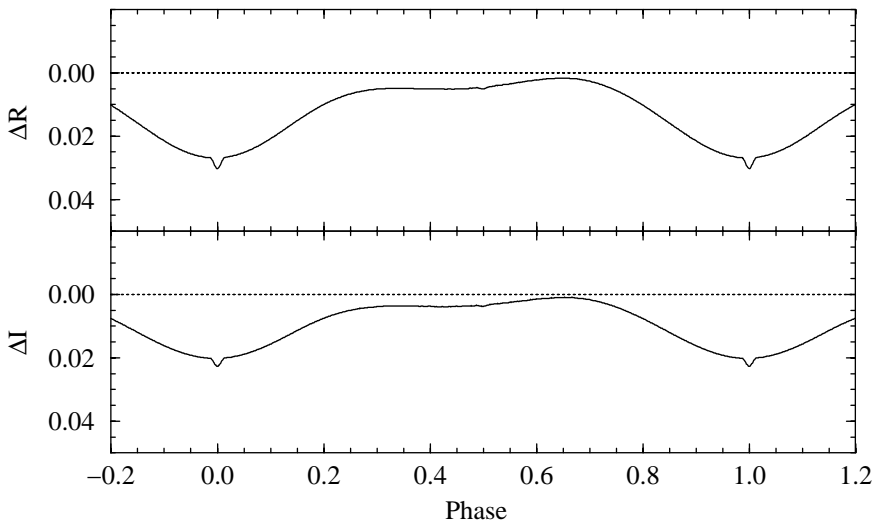

Fig. 2. Photometric effects of the spots on the $R$ and $I$ light curves. The spike during the phase of the primary minimum arises when the primary component is partially eclipsed and the spot on the secondary component is in front thus enhancing its relative contribution.

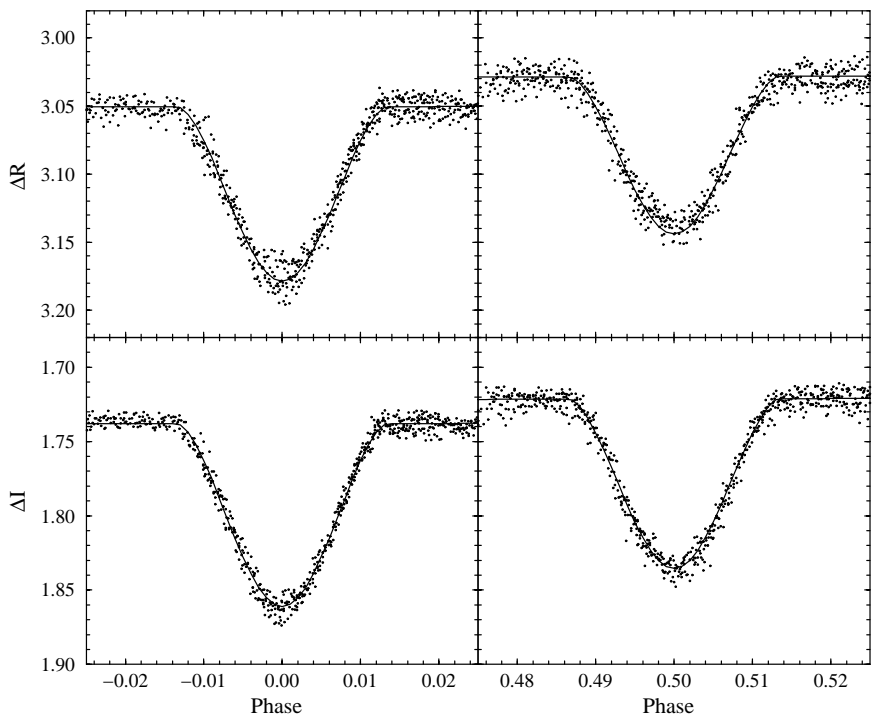

Fig. 3. Details of the fits to the primary and secondary eclipses. Note the high density of observations and the excellent agreement with the synthetic light curve.

and the geometry of the $\chi^{2}$ function near the minimum. The scatter in the resulting parameters from numerous additional solutions yielded estimated uncertainties that we consider to be more realistic, and are generally several times larger than the internal statistical errors. For CU Cnc, however, an additional error source must be considered and this is the uncertainty in the measurement of the luminosity ratio. The empirical value obtained from the spectra carries a small but significant measurement error that must be properly accounted for. We did this by running new W-D solutions for values of $\Omega_{A}$ set to yield the observed luminosity ratio plus and minus $1 \sigma$. The average differences between the adopted and the new parameters were combined quadratically with the uncertainties described above to yield the values listed in Table 3 . As a consequence of the uncertainty in the luminosity ratio, the radii of the components have larger errors $(\sim 2 \%)$ than would be expected from the quality of the light curve
Table 3. Results from the light curve analysis for CU Cnc.

\begin{tabular}{|c|c|}
\hline Parameter & Value \\
\hline \multicolumn{2}{|l|}{ Geometric and radiative parameters } \\
\hline$P$ (days) (fixed) & 2.771468 \\
\hline$e$ (fixed) & 0 \\
\hline$i(\operatorname{deg})$ & $86.34 \pm 0.03$ \\
\hline$q \equiv M_{\mathrm{B}} / M_{\mathrm{A}}($ fixed $)$ & 0.9184 \\
\hline$\Omega_{\mathrm{A}}($ fixed $)$ & $19.00 \pm 0.22$ \\
\hline$\Omega_{\mathrm{B}}$ & $19.39 \pm 0.40$ \\
\hline$r_{\mathrm{A}}($ volume $)$ & $0.0553 \pm 0.0007$ \\
\hline$r_{\mathrm{B}}($ volume $)$ & $0.0501 \pm 0.0012$ \\
\hline$k \equiv r_{\mathrm{B}} / r_{\mathrm{A}}$ & $0.906 \pm 0.025$ \\
\hline$T_{\mathrm{eff}}^{\mathrm{A}}(\mathrm{K})($ fixed $)$ & 3160 \\
\hline$T_{\mathrm{eff}}^{\mathrm{B}} / T_{\mathrm{eff}}^{\mathrm{A}}$ & $0.9892 \pm 0.0019$ \\
\hline$L_{\mathrm{B}} / L_{\mathrm{A}}(R$ band, phase 0.287$)$ & $0.74 \pm 0.05$ \\
\hline$L_{\mathrm{B}} / L_{\mathrm{A}}(I$ band, phase 0.287$)$ & $0.76 \pm 0.05$ \\
\hline$F_{3}(R$ band, phase 0.215$)$ (fixed) & 0.23 \\
\hline$F_{3}(I$ band, phase 0.215$)$ (fixed) & 0.25 \\
\hline Albedo (fixed) & 0.5 \\
\hline Gravity brightening (fixed) & 0.2 \\
\hline \multicolumn{2}{|c|}{ Limb darkening coefficients (Logarithmic law) } \\
\hline$x_{\mathrm{A}}$ and $y_{\mathrm{A}}(R$ band $)$ & $0.875,0.390$ \\
\hline$x_{\mathrm{B}}$ and $y_{\mathrm{B}}(R$ band $)$ & $0.875,0.390$ \\
\hline$x_{\mathrm{A}}$ and $y_{\mathrm{A}}(I$ band $)$ & $0.844,0.530$ \\
\hline$x_{\mathrm{B}}$ and $y_{\mathrm{B}}(I$ band $)$ & $0.844,0.530$ \\
\hline \multicolumn{2}{|l|}{ Spot parameters } \\
\hline Phase for spot \#1 & 0.438 \\
\hline Radius for spot \#1 (deg) & 9 \\
\hline$\Delta T$ for spot \#1 (K) & 450 \\
\hline Location of spot \#1 & Primary \\
\hline Phase for spot \#2 & 0.997 \\
\hline Radius for spot \#2 (deg) & 31 \\
\hline$\Delta T$ for spot \#2 (K) & 200 \\
\hline Location of spot \#2 & Secondary \\
\hline \multicolumn{2}{|l|}{ rms residuals from the fits } \\
\hline$\sigma_{R}(\mathrm{mag})$ & 0.008 \\
\hline$\sigma_{I}(\mathrm{mag})$ & 0.005 \\
\hline
\end{tabular}

The only independent determination of the light curve parameters of CU Cnc comes from D99. As mentioned earlier, the authors only had sparse observations and the quality of the solution is not very high. Importantly, the effect of star spots were taken into account in the analysis. The final radii published by D99 have errors of $12-16 \%$. Even so, the agreement between D99's solution and the parameters listed in Table 3 is mostly within their large quoted uncertainties. The most significant difference is in the relative radii of the components, where D99 report very unequal components $(k=0.67)$. Recall that the ratio of radii cannot be determined from the light curve alone so it is not surprising that D99 found rather inconsistent values. The sum of the relative radii, however, is tightly constrained by the duration of the eclipses and our value $(0.1054 \pm 0.0006)$ is extremely close to that found by D99 (0.1052). 
Table 4. Absolute dimensions and radiative properties for the components of CU Cnc.

\begin{tabular}{lll}
\hline \hline \multicolumn{1}{c}{ Property } & \multicolumn{1}{c}{ Primary } & \multicolumn{1}{c}{ Secondary } \\
\hline Spectral type & M3.5 Ve & M3.5 Ve \\
Mass $\left(M_{\odot}\right)$ & $0.4333 \pm 0.0017$ & $0.3980 \pm 0.0014$ \\
Radius $\left(R_{\odot}\right)$ & $0.4317 \pm 0.0052$ & $0.3908 \pm 0.0094$ \\
$\log g(\mathrm{cgs})$ & $4.804 \pm 0.011$ & $4.854 \pm 0.021$ \\
$T_{\text {eff }}(\mathrm{K})$ & $3160 \pm 150$ & $3125 \pm 150$ \\
$\log \left(L / L_{\odot}\right)$ & $-1.778 \pm 0.083$ & $-1.884 \pm 0.086$ \\
$M_{\text {bol }}(\mathrm{mag})$ & $9.19 \pm 0.21$ & $9.45 \pm 0.21$ \\
$\pi(\mathrm{mas})$ & \multicolumn{2}{c}{$78.05 \pm 5.69$} \\
$M_{V}(\mathrm{mag})$ & $11.95 \pm 0.16$ & $12.31 \pm 0.16$ \\
$B C_{V}(\mathrm{mag})$ & $-2.76 \pm 0.26$ & $-2.86 \pm 0.26$ \\
\hline
\end{tabular}

\section{Absolute dimensions and effective temperatures}

The absolute dimensions of the CU Cnc components can be readily determined by combining the photometric parameters in Table 3 with the spectroscopic solution from D99. When doing so, we obtain the physical properties listed in Table 4.

The absolute value of the effective temperature for eclipsing binaries cannot be determined from the light curves alone and independent methods must be used. Temperature determination is usually quite problematic when one has to deal with very cool stars such as CU Cnc. Two independent approaches present themselves to estimate the effective temperature of CU Cnc: one based on photometric calibrations and one based on the known absolute dimensions and distance.

Synthetic colors, which are the base of photometric calibrations, are computed by modelling the stellar atmospheres. Albeit some discrepancies remain, M-star model atmospheres have made significant progress over the past decade (Allard et al. 1997). Line opacities from a large number of molecular species are now included in the calculations and substantial improvements have been made in the input physics, particularly the equation of state. For cool stars, the infrared colors are especially suited to estimate the effective temperature because most of the stellar flux is emitted in that wavelength range. Thus, we collected from the literature the available magnitudes and colors for CU Cnc, which are listed in Table 5. Simple linear transformations were applied to refer the observed magnitudes and colors to the photometric systems most widely used.

The magnitudes and color indices in Table 5 correspond to the combined light of the two components of CU Cnc. Since the effective temperatures of the two components are very similar, it is justified to use the joint color indices as those representing the "mean" component of CU Cnc. To estimate the mean effective temperature we employed a number of modern photometric calibrations. We assumed no interstellar reddening in our calculations as expected for a system that lies less than $20 \mathrm{pc}$ away. The resulting temperatures, together with the references, atmosphere model names, and comments, are presented in Table 6. Most of the calibrations are based upon synthetic colors and model atmosphere calculations, except for Leggett (1992), who provides an empirical calibration. There is remarkably good agreement between all the temperature estimates. A plain average of the independent values (i.e., excluding one of the redundant temperatures from the BaSeL model) yields $T_{\text {eff }}=3140 \pm 40 \mathrm{~K}$. The formal error, however, does not reflect any possible systematics that could be present in the calibration. We have thus adopted a more realistic error value of $T_{\text {eff }}=3140 \pm 150 \mathrm{~K}$ based on the results of Leggett et al. (1996).

As mentioned above, there is an alternative approach to compute the temperature of the components based on the method described in Ribas et al. (1998). This is a simple method that relates the observed radius and luminosity with the temperature. In the case of $\mathrm{CU} \mathrm{Cnc}$, the absolute radii of the components are given in Table 4. The empirical luminosities were computed from the apparent magnitudes, the parallax in the Hipparcos catalogue (ESA 1997) and a bolometric correction. The latter is especially delicate because bolometric correction calibrations are known to be the source of systematic errors. To minimise these, we used the bolometric correction in the $K$ band, which was computed from the models of Allard et al. (2000) and resulted in $B C_{\mathrm{KBB}}=+2.75 \pm$ $0.08 \mathrm{mag}$. This leads to an apparent bolometric luminosity of $m_{\mathrm{bol}}=9.42 \pm 0.08$. From the luminosity ratio, the individual bolometric magnitudes can be computed and we find $m_{\text {bol A }}=10.04 \pm 0.08$ and $m_{\text {bolB }}=10.31 \pm 0.08$. The absolute bolometric magnitudes follow from introducing the Hipparcos distance: $M_{\mathrm{bolA}}=9.50 \pm 0.18$ and $M_{\mathrm{bolB}}=9.77 \pm 0.18$. The effective temperature of each component can be computed in a straightforward manner and we obtain $T_{\text {eff A }}=2950 \pm 120 \mathrm{~K}$ and $T_{\text {eff B }}=2910 \pm 120 \mathrm{~K}$.

The agreement with the photometric determinations described above is not perfect but nevertheless fairly good given the uncertainties. The values agree within one sigma of their respective error bars. However, a caveat is due at this point. As it turns out, there are reasonable concerns about the accuracy of the Hipparcos parallax, even taking into account its relatively large error (see Table 4). CU Cnc is a faint object near the threshold for detection by Hipparcos. Thus, the individual transit data are of poor quality. A reanalysis of the transit data (considering also the visual component) has not provided any better results (Arenou 1999, priv. comm.). As a reference, we have computed the temperature of $\mathrm{CU} \mathrm{Cnc}$ as a function of the distance $^{3}$ :

$$
T_{\text {eff A }}=2610 \sqrt{\frac{d(\mathrm{pc})}{10}} \mathrm{~K} ; \quad T_{\text {eff B }}=2575 \sqrt{\frac{d(\mathrm{pc})}{10}} \mathrm{~K} .
$$

Given the concerns with the Hipparcos distance raised above, we prefer to adopt the temperatures derived from photometric calibrations. The final adopted individual temperatures for CU Cnc (computed from the average temperature and the temperature ratio derived from the light curves) are listed in Table 4 along with their associated errors. Also included in Table 4 are the absolute magnitudes of the components in the $V$ band, which have been computed from the parallax listed in the Hipparcos catalogue. Note that an empirical determination of

\footnotetext{
3 Note that there is a weak dependence of the bolometric correction on the temperature, so these expressions are only valid for temperatures not too far off from those in Table 4.
} 
Table 5. Joint observed magnitudes and color indices for CU Cnc.

\begin{tabular}{lll}
\hline \hline Mag. or color & \multicolumn{1}{c}{ Value } & \multicolumn{1}{c}{ Notes } \\
\hline$V$ & $11.89 \pm 0.01$ & From Weis (1991) \\
$(R-I)_{\mathrm{C}}^{\mathrm{a}}$ & 1.60 & From Weis (1991) and transformations in Bessell (1979) \\
$(V-I)_{\mathrm{C}}^{\mathrm{a}}$ & 2.80 & From Weis (1991) and transformations in Bessell (1979) \\
$J_{2 \text { MASS }}$ & $7.536 \pm 0.018$ & 2MASS Second Incremental Data Release \\
$H_{2 \mathrm{MASS}}$ & $6.912 \pm 0.030$ & 2MASS Second Incremental Data Release \\
$K_{2 \mathrm{MASS}}^{\mathrm{b}}$ & $6.626 \pm 0.029$ & 2MASS Second Incremental Data Release \\
$K_{\mathrm{BB}}^{\mathrm{c}}$ & $6.670 \pm 0.031$ & Using transformations from Carpenter (2001) \\
$K_{\mathrm{CIT}}^{\mathrm{d}}$ & $6.650 \pm 0.031$ & Using transformations from Carpenter (2001) \\
$(V-K)_{\mathrm{BB}}^{\mathrm{c}}$ & $5.22 \pm 0.03$ & \\
$(V-K)_{\mathrm{CIT}}^{\mathrm{d}}$ & $5.24 \pm 0.03$ & \\
\hline
\end{tabular}

a Cousins (1976) photometric system.

b http://www.ipac.caltech.edu/2mass/releases/second/doc/explsup.html

c Bessell \& Brett (1988) homogenized system.

d Caltech photometric system (Elias et al. 1982, 1983).

Table 6. Effective temperature determinations for the mean component of CU Cnc.

\begin{tabular}{lccl}
\hline \hline \multicolumn{1}{c}{ Reference } & Model & $T_{\text {eff }}(\mathrm{K})$ & \multicolumn{1}{c}{ Colors used } \\
\hline Allard et al. (2000) & STARDusty2000 & 3130 & $(V-I)_{\mathrm{C}},(V-K)_{\mathrm{CIT}}$ \\
Hauschildt et al. (1999) & NextGen & 3170 & $(V-K)_{\mathrm{CIT}}$ \\
Lejeune et al. (1998) & BaSeL 3.1 & 3170 & $(V-I)_{\mathrm{C}},(V-K)_{\mathrm{BB}}$ \\
Lejeune et al. (1998) & BaSeL 2.2 & 3170 & $(V-I)_{\mathrm{C}},(V-K)_{\mathrm{BB}}$ \\
Bessell et al. (1998) & NMARCS & 3070 & $(V-I)_{\mathrm{C}},(V-K)_{\mathrm{BB}}$ \\
Leggett (1992) & - & 3145 & $(R-I)_{\mathrm{C}},(V-I)_{\mathrm{C}},(V-K)_{\mathrm{CIT}}$ \\
\hline
\end{tabular}

the bolometric correction for each component follows from the comparison of the $V$-band absolute magnitude with the bolometric magnitudes (resulting from the radii, effective temperatures and $\left.M_{\text {bol } \odot}=4.74\right)$.

\section{Other observational information}

\subsection{Age and chemical composition}

One of the goals of the present study is to carry out a critical analysis of low-mass stellar models similar to that in TR02. Thus, to make the test as stringent as possible, one has to keep the number of degrees of freedom at a minimum. Two of the key parameters that determine the observable physical properties of a star are its age and chemical composition (to simplify, metallicity). The metallicity of a star is generally determined through high-resolution spectroscopy and comparison with synthetic spectra. Unfortunately, this is virtually impossible for M stars because of the huge quantity of spectral features and the shortcomings of the atmosphere models. In turn, the age (and eventually the metallicity) can only be derived if the star belongs in a well-studied cluster. Field M stars are, thus, not ideally suited for reliable estimations of age and metallicity.

One possible way to obtain a rough estimation of the age and chemical composition of a field M star such as CU Cnc is through the analysis of its space velocities. High velocity would likely indicate an (old) halo population and low velocity a (young) star in the galactic disk. The space velocities $(U, V, W)$ of a star are readily computed from its position, proper motions, radial velocity and distance. In the case of $\mathrm{CU} \mathrm{Cnc}$, we have adopted the Hipparcos position, proper motion and distance, and the radial velocity has been taken from D99. The resulting space velocities are $U=-10.7 \mathrm{~km} \mathrm{~s}^{-1}, V=-4.9 \mathrm{~km} \mathrm{~s}^{-1}$, and $W=-10.6 \mathrm{~km} \mathrm{~s}^{-1}$. Note that we have followed the convention where positive values of $U, V$, and $W$ indicate velocities towards the galactic center, galactic rotation and galactic North Pole, respectively.

The low values of these velocities are an indication of a disk population and thus a relatively young star. A closer look at the values revealed that these are strikingly similar to the velocities of the Castor moving group (see Anosova \& Orlov 1991; Barrado y Navascués 1998) of which YY Gem is a likely member. Ribas (2003) utilised various criteria (isochrones, rotationactivity relationships, etc.) to estimate an age of $320 \pm 80 \mathrm{Myr}$ and a metal content of $Z=Z_{\odot}$ for the moving group members. The mean space velocities of the moving group have been recently re-determined by Ribas (2003) to be $\langle U\rangle=$ $-10.6 \pm 3.7 \mathrm{~km} \mathrm{~s}^{-1},\langle V\rangle=-6.8 \pm 2.3 \mathrm{~km} \mathrm{~s}^{-1}$, and $\langle W\rangle=$ $-9.4 \pm 2.1 \mathrm{~km} \mathrm{~s}^{-1}$. These are indeed very similar to the values found for CU Cnc, which strongly suggests its membership of the Castor moving group. Thus, it is sensible to assume that CU Cnc has the same age and chemical composition as the rest of the group members - similarly to what is routinely done in stellar cluster studies. As it happened with YY Gem (TR02) the fortunate fact that CU Cnc belongs in the moving group and has a well-determined age and metal content greatly enhances the value of the star since it allows for an unusually stringent test of the theoretical models. 


\subsection{Lithium abundance}

As it has been known for some time, the measured abundance of lithium in the stellar atmosphere can be used as an age indicator for young cool stars, particularly in clusters (e.g., Duncan 1981; Soderblom et al. 1990). Theoretical studies demonstrate that the ${ }^{7} \mathrm{Li}$ nuclei are destroyed by proton collisions at temperatures above 2.5 MK (Bodenheimer 1965; D'Antona \& Mazzitelli 1984). In low mass stars, mixing through convection processes is so efficient that $\mathrm{Li}$ is depleted at a very rapid pace. We have inspected our high-resolution spectrum described in Sect. 3 and we report a tentative detection of weak Li I $\lambda 6708$ features at the expected wavelengths for both the primary and secondary components. The equivalent width measurement (corrected for light dilution) yields a value of $\sim 50 \mathrm{m \AA}$ for both stars. From this and the theoretical study of Pavlenko et al. (1995), one can roughly estimate the $\mathrm{Li}$ abundance to be $\log N(\mathrm{Li}) \approx-1.1$ (in the scale in which $\log N(\mathrm{H})=12$ ), and $\log \left[n(\mathrm{Li}) / n_{\circ}(\mathrm{Li})\right]=-4.1$. Thus, the initial $\mathrm{Li}$ content of CU Cnc's components appears to have been mostly destroyed, yet not completely. This is in apparent contradiction with $\mathrm{Li}$ destruction sequences in clusters and associations (Barrado y Navascués et al. 1999; Stauffer et al. 1999), which indicate that mid-M type stars fully deplete their initial Li abundance in as little as a few times $10^{7}$ years. (Recall that the estimated age for CU Cnc is 320 Myr.) Barrado y Navascués et al. (1997) observed a similar discrepancy with the observed Li abundance $(\log N(\mathrm{Li})=0.0)$ for the eclipsing binary YY Gem. In this case, Li also appears to be severely depleted but not fully destroyed, as would be expected for YY Gem's age of 320 Myr. If we rely on the ages determined for these binaries, the two results seem to indicate that $\mathrm{Li}$ depletion for $\mathrm{M}$ stars in binary systems might be not as efficient as for single stars. For example, orbital synchronization due to tidal forces could be responsible for inhibiting turbulent mixing, thus slowing down Li depletion (Barrado y Navascués et al. 1997). The tentative detection of $\mathrm{Li}$ in the spectrum of $\mathrm{CU} \mathrm{Cnc}$ is a puzzle yet to be resolved a detailed discussion of this topic is left for an upcoming work.

\subsection{Stellar activity}

Other observational information for CU Cnc can be inferred from the high-resolution spectrum described in Sect. 3. An inspection of the spectrum reveals very strong $\mathrm{H} \alpha$ and $\mathrm{H} \beta$ emission features (double lines). The measurements yield (continuum-corrected) $\mathrm{H} \alpha$ equivalent widths of $3.85 \AA$ and $4.05 \AA$ for the primary and secondary components, respectively. For comparison, Young et al. (1989) report an excess equivalent width of $\approx 2 \AA$ for the presumably coeval but more massive system YY Gem. The $\mathrm{H} \alpha$ equivalent widths of both CU Cnc and YY Gem are rather large but not unreasonable when compared with young M-type stars (see, e.g., Soderblom et al. 1991). Note, however, that the strong $\mathrm{H} \alpha$ emission in these binary systems is more related to the spin-up caused by orbital synchronization rather than age.

In addition to displaying $\mathrm{H} \alpha$ chromospheric emission, $\mathrm{CU} \mathrm{Cnc}$ is also both and EUV and X-ray source. From the observations in the ROSAT All-Sky Bright Source Catalogue
(Voges et al. 1999) and the calibration in Schmitt et al. (1995) one obtains an integrated X-ray luminosity of $\log L_{X}\left(\mathrm{erg} \mathrm{s}^{-1}\right)=$ 29.03 for CU Cnc. Again, this value can be compared with YY Gem's, which was estimated to be $\log L_{\mathrm{X}}\left(\mathrm{erg} \mathrm{s}^{-1}\right)=29.61$ (Güdel et al. 1993). One must keep in mind that the size of the stars in CU Cnc is significantly smaller than those of YY Gem. Thus, the ratio $L_{\mathrm{X}} / L_{\mathrm{bol}}$ is a more realistic measure of the stellar activity or, in other words, of the efficiency in producing high energy emissions. The calculations of $L_{\mathrm{X}} / L_{\mathrm{bol}}$ yield a value very close to $10^{-3}$ for both CU Cnc and YY Gem, thus indicating very similar activity levels. Numerous studies (see, e.g., Jeffries \& Tolley 1998; Gagne et al. 1995) suggest that this value of $L_{\mathrm{X}} / L_{\mathrm{bol}}=10^{-3}$ indeed marks a saturation limit in the stellar activity.

\section{Critical comparison with stellar models}

$\mathrm{CU}$ Cnc is only the third M-type eclipsing binary for which accurate absolute dimensions (masses and radii) have been determined. In addition, we have been able to set rather stringent constraints on the effective temperatures of the components as well as on the age and chemical composition of the system. With this information in hand, we are in a position to carry out a critical evaluation of the stellar model predictions. For the first time we can provide observational checks on M stars of about $0.4 M_{\odot}$ for which no reliable empirical information has been available to date. The procedure followed here is very similar to that described in TR02 for YY Gem, but with a significant difference. While the two components of YY Gem are almost identical, this is not the case for CU Cnc where the components differ by almost $10 \%$ in mass. Since the two stars in the binary system are coeval, a single isochrone expected to fit both components simultaneously. This implies that not only the position in the observational diagrams but also the slopes of the isochrones come into play. Thus, the models must reproduce both the absolute and the relative location of the components.

For our comparison we have considered nine different sets of theoretical calculations: Swenson et al. (1994), D'Antona \& Mazzitelli (1997), Siess et al. (1997), Baraffe et al. (1998), Palla \& Stahler (1999), Charbonnel et al. (1999), Girardi et al. (2000), Yi et al. (2001), and Bergbusch \& VandenBerg (2001). These include essentially all of the modern models for low mass stars, even though some of them do not quite reach down to the masses of CU Cnc. We have, nevertheless, incorporated them so that their performance at higher masses can be compared with the rest of the models. Isochrones for an age of $320 \mathrm{Myr}$ and $Z=0.018$ have been interpolated for all models except those by Palla \& Stahler (1999), with an oldest age available of $100 \mathrm{Myr}$ (this has no effect whatsoever on our conclusions). The models of Siess et al. (1997) and Baraffe et al. (1998) employ the most sophisticated physics (equation of state) and boundary conditions (non-grey model atmospheres), so, in principle, they would be expected to provide the best fit to the physical properties of CU Cnc.

We have compared the observational data for $\mathrm{CU} \mathrm{Cnc}$ with the model predictions at different planes. The plots are shown in the eight panels of Fig. 4. For clarity reasons, we have plotted the models of Siess et al. (1997) and Baraffe et al. (1998) 

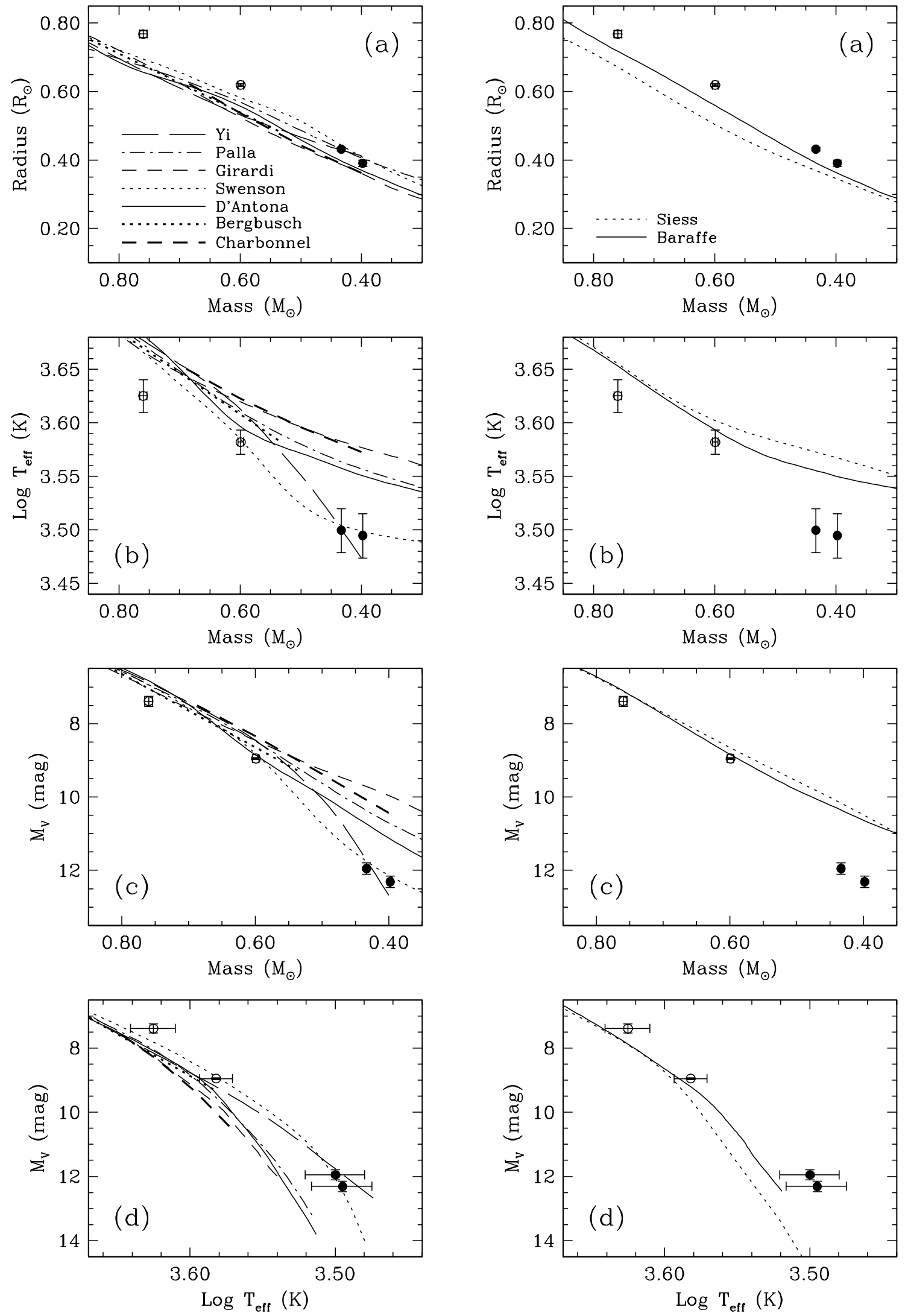

Fig. 4. The location of CU Cnc's components on several observational planes is compared with isochrones from nine different theoretical models (see legends and references in text). The age for the isochrones is $320 \mathrm{Myr}$ and the metallicity adopted is $Z=0.018$, except for the isochrones by Palla \& Stahler (1999) that are for an age of $100 \mathrm{Myr}$ and solar abundance. In addition to the components of CU Cnc (represented with filled circles), we show the locations of YY Gem's mean component at a mass of $0.6 M_{\odot}$ and the secondary component of V818 Tau with a mass of $0.76 M_{\odot}$ (both represented as open circles). 
separated from the rest in the right panels. Note that we have also included the observational data on YY Gem AB and V818 Tau B (both from TR02), which will help to understand the comparison in a wider context. The best determined parameters of our analysis are the masses and the radii, which are purely empirical, whereas the effective temperatures and absolute magnitudes hinge to some extent on external calibrations. Thus, the mass-radius diagram in Fig. 4a constitutes the most reliable check on the models, also because the error bars of the measurements are very small. As can be seen, some of the models yield reasonably good, albeit not perfect, fits to the data. The three isochrones that exhibit the best performance are those by Swenson et al. (1994), Palla \& Stahler (1999), and Yi et al. (2001). However, among these, only the models of Swenson et al. (1994) succeed in reproducing at the same time the relative location (i.e. slope) of the binary components. The models of Siess et al. (1997) and Baraffe et al. (1998), in spite of using state-of-the-art physical ingredients, do not yield a good fit to the data, although the latter perform slightly better. The radii that these models predict at the CU Cnc's masses are about 10$14 \%$ smaller than observed. (Note that this difference amounts to $\sim 7 \sigma$.) The plots indicate that the huge discrepancies between model predictions and observations encountered by TR02 at slightly higher masses (YY Gem AB and V818 Tau B) are not quite as dramatic at the mass regime of $\mathrm{CU} \mathrm{Cnc}$, and some of the models produce reasonable fits to the data.

The panels in Fig. $4 \mathrm{~b}$ introduce the effective temperature in the comparison. Here the internal discrepancies among the seven models grow larger as we move towards lower masses. At around $0.4 M_{\odot}$ differences of up to $20 \%$ exist in the effective temperatures predicted by the different isochrones. Interestingly, most of the models seem to indicate temperatures that are significantly (10-15\%) higher than observed for CU Cnc. The isochrones of Swenson et al. (1994) and Yi et al. (2001) clearly stand out among all and appear to produce a fairly close fit. But again, only the isochrone computed from the models by Swenson et al. (1994) reproduces the relative location of the components to achieve an outstanding fit to CU Cnc and also YY Gem, although V818 Tau B seems significantly cooler than model predictions.

The mass-luminosity diagram in Fig. 4c presents a situation which is analogous to that described above. Most of the isochrones predict $M_{V}$ 's for CU Cnc that are too bright by up to 2 mag. For example, the models of Siess et al. (1997) and Baraffe et al. (1998) yield close fits to both V818 Tau B and YY Gem while huge differences are found with the observed magnitudes of CU Cnc. The models by Swenson et al. (1994) are able to both reproduce the absolute magnitude of CU Cnc and YY Gem, while also achieving a quite close fit to V818 Tau B. At the same time, the slope of the isochrone matches perfectly the relative configuration of the CU Cnc components.

Finally, the H-R diagrams in Fig. 4d do not reserve any surprises. Most of the models tend to overestimate the effective temperature at a given absolute magnitude. The best agreement is found for the models of Swenson et al. (1994) that produce an isochrone with the correct slope that reproduces well the observed parameters of CU Cnc and YY Gem. Note that the relatively close fit by the models of Baraffe et al. (1998) is fictitious because it occurs for masses that are far off from those determined for CU Cnc.

\section{Is CU Cnc akin to other stars of similar mass?}

The comparison discussed above leaves no doubt that most of current stellar models fail in reproducing the observed physical properties of CU Cnc. Only the models by Swenson et al. (1994) yield reasonably good (even excellent) isochrone fits to the data. Even though $\mathrm{CU} \mathrm{Cnc}$ is the only object with a mass around $0.4 M_{\odot}$ with fully characterised physical properties, there are other stars in this regime whose masses have been spectroscopically determined to good accuracy. This is the case of Gl 570C, Gl 623A, Gl 644A, Gl 644Ba, Gl 661A, and G1 661B, all with masses between 0.34 and $0.42 M_{\odot}$ (see Forveille et al. 1999; Ségransan et al. 2000; Delfosse et al. 2000; Martin et al. 1998; Mazeh et al. 2001). We have compiled $V$ and $K$ absolute magnitudes for these stars from Delfosse et al. (2000). Two mass-absolute magnitude plots are shown in Fig. 5, one for the $V$ magnitude and one for the $K$ magnitude. The results are quite striking as $\mathrm{CU}$ Cnc appears to be fainter in both plots than other stars of the same mass. The magnitude difference is about $1.4 \mathrm{mag}$ in the $V$ band and $0.35 \mathrm{mag}$ in the $K$ band. The apparent faintness of $\mathrm{CU} \mathrm{Cnc}$ in the $V$ band had already been pointed out by D99, Delfosse et al. (2000) and Mazeh et al. (2001). Note that the isochrones computed with the models of Baraffe et al. (1998) and Siess et al. (1997) plotted in the figure produce a reasonably close fit to the stars other than CU Cnc in both observational diagrams. On the other hand, the isochrone by Swenson et al. (1994) - only available in $M_{\mathrm{V}}-$, which closely matches $\mathrm{CU} \mathrm{Cnc}$, fails in reproducing the observed absolute magnitudes of the other stars.

Let us carefully analyse possible reasons for the discrepancy. One scenario to explain the disagreement is the effect of enhanced stellar activity in CU Cnc due to binarity (higher rotational velocity). This would cause the appearance of large spots on the surface of the stars and also an increase of the $\mathrm{TiO}$ absorption. It is well known that the $V$ passband is strongly affected by $\mathrm{TiO}$ absorption whereas the $K$ band is mostly free from TiO bands. This might cause the star to appear especially faint in the $V$ band. There are, however, evidences that argue against this scenario. All observational data (X-ray luminosity, $\mathrm{H} \alpha$ emission) indicate that $\mathrm{CU} \mathrm{Cnc}$ is not especially active when compared with YY Gem, which is quite closely matched by the models. At CU Cnc's mass range, the ROSAT All-Sky Bright Source Catalogue (Voges et al. 1999) yields an estimated X-ray luminosity for the stars in the Gliese 644 system (plotted in Fig. 5) of $\log L_{X}\left(\mathrm{erg} \mathrm{s}^{-1}\right)=29.14$, thus indicating an activity level very similar to CU Cnc's. In addition, the available multi-band photometry (see Table 5) refutes the idea that the apparent faintness of CU Cnc is only restricted to the $V$ band. The spectral energy distribution deduced from the photometric data is internally consistent, i.e. all photometric indices yield the same effective temperature. Thus, it is the temperature of $\mathrm{CU} \mathrm{Cnc}$ and not the photometry the source of the discrepancy. Using $(V-K)$ indices and the calibrations in Sect. 4, we estimate that $\mathrm{CU} \mathrm{Cnc}$ is some $300 \mathrm{~K}$ cooler than other stars of about $0.4 M_{\odot}$. That the mean photospheric 


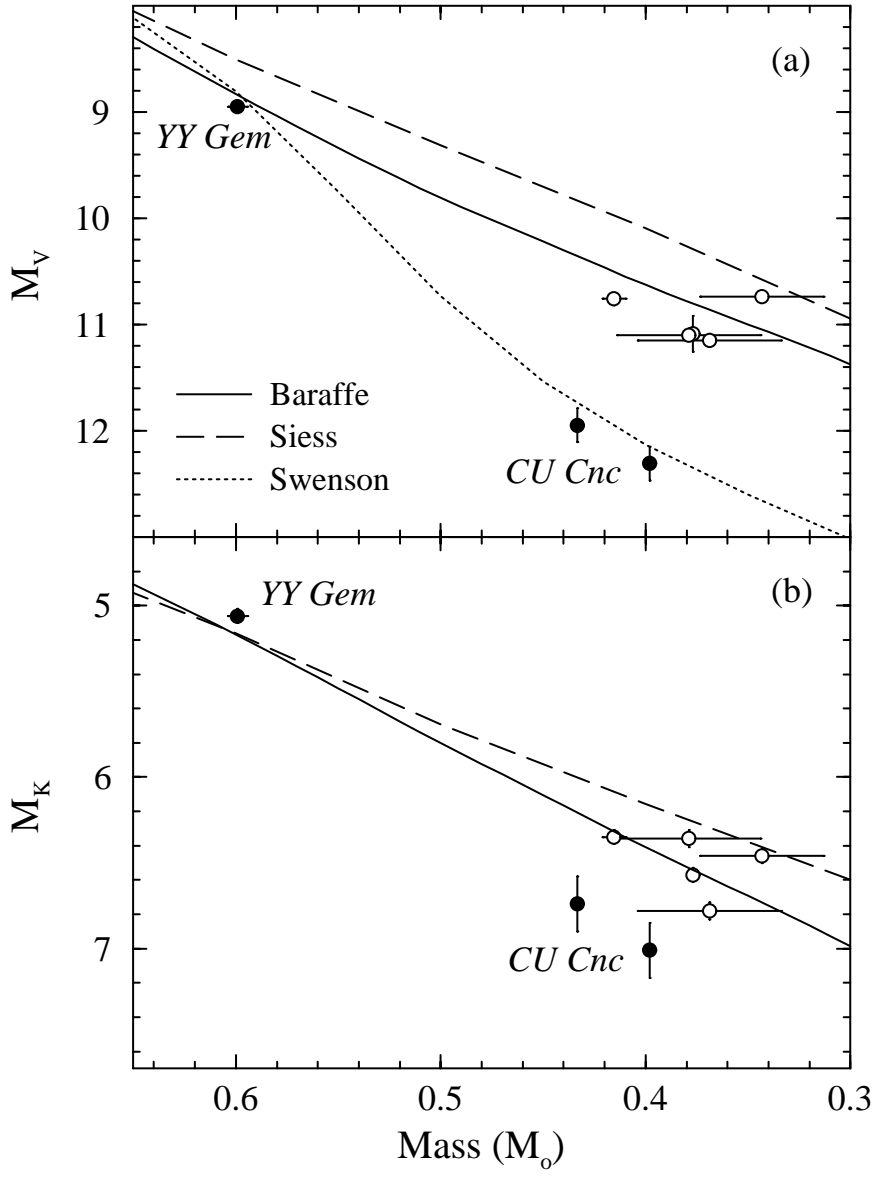

Fig. 5. Mass-absolute magnitude diagrams in the $V$ and $K$ bands for the lower main sequence. CU Cnc is shown together with YY Gem and other stars with spectroscopically-determined masses (see text). The lines represent isochrones for the estimated age of $\mathrm{CU}$ Cnc computed with different models.

temperature is reduced by as much as $10 \%$ due to enhanced stellar activity in $\mathrm{CU}$ Cnc seems, in principle, quite unlikely. More so if we take into account that stellar activity produces photospheric plages in addition to dark spots that would (partly or totally) compensate for the temperature reduction.

The distance to $\mathrm{CU} \mathrm{Cnc}$ is another possible source for discrepancies since, as we have discussed in Sect. 4, it might be subject to systematic errors. However, a magnitude differential in the distance modulus of the star would be constant over wavelength and affect identically both $V$ and $K$ bands. This is a strong argument against this scenario because we observe a much larger deviation in $M_{V}$ than in $M_{K}$. Nonetheless, a problem with the distance could still be responsible for a fraction of the discrepancy.

Our estimate for the metal abundance for $\mathrm{CU} \mathrm{Cnc}$ comes from its likely membership of the Castor moving group. The possibility for a different chemical composition can be not completely discarded because the star might not belong in the group after all or have different abundances than other group members. A very high metal abundance of $[\mathrm{Fe} / \mathrm{H}]=+0.5$ was put forward by D99 as a possible explanation for the anomalous location of CU Cnc in the mass-absolute magnitude diagram. This was proposed on the basis of the isochrone behaviour in the mass $-M_{V}$ diagram for metal abundances of $[\mathrm{Fe} / \mathrm{H}]=0$ and
$[\mathrm{Fe} / \mathrm{H}]=-0.5$. We have carried out this analysis again with the new parameters for $\mathrm{CU} \mathrm{Cnc}$ and the models of Baraffe et al. (1998). If, as assumed by D99, the absolute magnitude changes are supposed to scale linearly with metallicity, a value even higher than +0.5 is necessary since the $V$ magnitude difference between $[\mathrm{Fe} / \mathrm{H}]=0$ and $[\mathrm{Fe} / \mathrm{H}]=-0.5$ at a mass of $0.4 M_{\odot}$ is only 0.8 mag. We recall that $\mathrm{CU}$ Cnc appears to be dimmer by as much as $1.4 \mathrm{mag}$. Furthermore, this scenario does not explain the discrepancy in the $K$ band because the different metallicity isochrones run virtually on top of one another. An additional argument against the high-metallicity hypothesis is the lack of other stars in the solar neighbourhood with such high metal abundance. In the thorough study of Edvardsson et al. (1993) the highest metallicity found was $[\mathrm{Fe} / \mathrm{H}]=+0.24$. The catalog of Cayrel de Strobel et al. (2001) lists only a handful of stars with $[\mathrm{Fe} / \mathrm{H}]>+0.4$ and most of them appear to be misclassified. The high metallicity proposed to resolve the issue with the location of CU Cnc in the mass- $M_{V}$ diagram seems, at this point, quite unlikely.

A younger age than estimated for $\mathrm{CU} \mathrm{Cnc}$ from its membership of the Castor moving group would neither explain the discrepancy. A low mass star becomes dimmer as it evolves from the pre-main sequence phase towards the ZAMS, where the minimum luminosity is reached. CU Cnc is already fainter than the other stars in the same mass range. Alternatively, it could be that the stars used for comparison with CU Cnc (Gl 570, Gl 623, Gl 644, Gl 661) were all much younger ( 10-20 Myr) and contracting towards the ZAMS. Once again, this hypothetical situation is not in agreement with the observed massmagnitude diagrams because the magnitude change in the $V$ and $K$ bands are predicted by the models to be very similar (actually, a somewhat larger magnitude variation in the $K$ band).

Thus far, there are no plausible scenarios to account for the observed magnitude differences between CU Cnc and similar mass stars. Even though it might appear as improbable, the presence of a certain amount of dust absorption could reproduce the observed behaviour. Indeed, according to Fitzpatrick (1999), the relationship between $A_{V}$ and $A_{K}$ for interstellar dust is $A_{V} \approx 8 A_{\mathrm{K}}$. Thus, 1.4 mag of extinction in $V$ would roughly correspond to $0.2 \mathrm{mag}$ in $K$. Such magnitude corrections would bring the two mass-magnitude plots into fair agreement. At a distance of only $\sim 13 \mathrm{pc}$, it can be definitely ruled out that lineof-sight interstellar dust could account for over 1 mag of extinction in the $V$ band $(E(B-V)=0.42)$. The alternative is therefore circumstellar dust. Disks of circumstellar dust have been directly detected in a number of nearby stars (see, e.g., Greaves et al. 1998 and references therein) and some of them have estimated ages similar to CU Cnc's. If the disk was coplanar with the binary's orbit, as it might likely be assumed, we could be observing the stars through a line of sight with enhanced dust absorption. There are no observational grounds to support such hypothesis yet but, at this point, this is the only scenario that explains the observations.

If the disk hypothesis is proven to be correct, our temperature estimate (which is based on multi-colour photometry) and thus the comparison with stellar models in the temperature and absolute magnitude diagrams would have to be revised accordingly. A rough estimate made by correcting for the differences 
of $1.4 \mathrm{mag}$ in $V$ and $0.35 \mathrm{mag}$ in $K$ raises the temperature of the components to $T_{\text {eff A }}=3440 \pm 150 \mathrm{~K}$ and $T_{\text {eff }}=3400 \pm 150 \mathrm{~K}$. Also, the effective temperatures obtained from the radii, the corrected apparent magnitudes and the Hipparcos parallax (see Sect. 4) are $T_{\text {eff A }}=3290 \pm 140 \mathrm{~K}$ and $T_{\text {eff B }}=3240 \pm 140 \mathrm{~K}$, thus achieving a remarkable 1 sigma agreement with the photometric values.

\section{Conclusions}

The lack of observational constraints is one of the most severe deficiencies that stellar models for the lower main sequence have to face. Eclipsing binaries are thus crucial because they yield simultaneous determinations of masses and radii (and also temperatures) upon which the stellar models can be checked. We have therefore acquired and analysed highquality $R$ and $I$ light curves with very dense phase coverage (over 2100 observations) of the low-mass eclipsing binary CU Cnc. In the light curve analysis, carried out with the W-D program, we resolved an inherent indeterminacy of the ratio of radii of the components (caused by the partial eclipses) by fixing the luminosity ratio to the value obtained from a high-resolution spectrum. Also, special care has been put in modelling the conspicuous out-of-eclipse variations through surface inhomogeities. The orbital and physical properties resulting from the light curves were combined with the spectroscopic parameters from D99 to achieve precisions better than $2 \%$ in the absolute dimensions of CU Cnc components. As a result of this, CU Cnc is the third system (together with YY Gem and CM Dra) to reach sufficient accuracy in its absolute dimensions so that critical tests of stellar models can be performed.

To make the comparison with models more stringent, we made use of further observational constraints. For example, the mean effective temperature of CU Cnc was determined from multi-band photometry (optical through infrared) and synthetic colours resulting from state-of-the-art atmosphere models. Additionally, the space motions of CU Cnc strongly suggest membership of the Castor moving group. This allowed us to infer rough values for the age of the star $(\sim 320 \mathrm{Myr})$ and its chemical composition $(Z \approx 0.018)$. With this host of observational information - quite uncommon for most eclipsing binaries -, we carried out a detailed comparison with all of the recent low-mass stellar models. First, the most reliable check is in the mass-radius diagram because these are two quantities empirically determined from our analysis. The comparison indicates that, whereas some of the models provide radius values that are in reasonable agreement with the observations, the most sophisticated models by Siess et al. (1997) and Baraffe et al. (1998) yield radii that are some 10\% smaller than observed. The study of three additional observational planes indicates that CU Cnc's components are also significantly cooler $(\sim 15 \%)$ and fainter in the $V$ band $(\sim 1.5 \mathrm{mag})$ than predicted by most models.

A matter of some concern arises when comparing CU Cnc with other stars of similar mass. The comparison indicates that CU Cnc is some $1.4 \mathrm{mag}$ fainter in the $V$ band and $0.35 \mathrm{mag}$ fainter in the $K$ band. We have analysed a number of possible scenarios to explain the discrepancy and ruled out most of them on the basis of the available observational information. For example, we have discarded the effect of stellar activity, an error in the distance, a different age or an extreme chemical composition. Only two scenarios appear to succeed in explaining the observed differences in the mass-magnitude diagrams: 1) CU Cnc's components are, for a yet unknown reason, about $10 \%$ cooler than other stars of the same mass, and 2) there is a certain amount of (circumstellar) dust absorption that is responsible for the reddening of the spectral energy distribution. If the latter turns out to be the correct scenario, this is a very exciting result. Given the almost edge-on configuration of the eclipsing binary, we could be observing CU Cnc through a coplanar dusty disk. Definite proof of this should come from submillimeter observations where the thermal emission of the dust could be detected. Note that CU Cnc belongs in the same moving group and probably has similar age to Vega and Fomalhaut (Barrado y Navascués 1998), both stars with bona-fide circumstellar dust disks (Holland et al. 1998). With the tentative detection of a Vega-like edge-on dust disk and its relatively low mass and surface brightness, CU Cnc also becomes a very interesting target for planet searches through radial velocity studies or the transit method.

In our comparative study, we have concluded that the evolutionary models of Swenson et al. (1994) clearly stand out among all because they achieve the best performance in reproducing all of the observed properties of CU Cnc and even the relative position of the components (much better constrained from the light curves). However, these models cannot obviously fit the position of other $0.4-M_{\odot}$ stars in the massabsolute magnitude diagrams, whereas models such as Siess et al. (1997) and especially Baraffe et al. (1998) provide more reasonable fits. Unfortunately, until the discrepancy between the radiative properties of $\mathrm{CU} \mathrm{Cnc}$ and stars of similar mass is resolved, no strong constraints on the models can be set in the temperature and absolute magnitude diagrams. A better determination of the parallax and an empirical estimation of the metal abundance would surely help to clarify the situation.

Regardless of the issue with the temperature, the comparison of CU Cnc's absolute dimensions (empirically-measured masses and radii) with stellar model predictions clearly confirms the trend seen by TR02 for YY Gem and V818 Tau. The systematic underestimation of the radii of the stars can lead to severely underestimated stellar ages when using the $\mathrm{H}-\mathrm{R}$ diagram diagnostics. In spite of recent advances, it seems clear that further adjustments are needed in evolution models for the lower main sequence to achieve a good description of the observed stellar physical properties.

Note added in proofs: Forveille (2002, priv. comm.) has recently pointed out that adaptative optics observations reveal a faint close companion to $\mathrm{CU} \mathrm{Cnc}$ at a distance of $00^{\prime} 68$. The measurements by Beuzit at al. (2001, [astro-ph/0106277]) yield a magnitude difference in the $K$ band of 3.2 mag between the CU Cnc system and the companion. This detection has an impact, albeit limited, on the results presented in this paper. Firstly, an unresolved companion could very well be responsible for a poor parallax determination by Hipparcos, 
thus explaining the discrepancies discussed in Sect. 4 . Secondly, new calculations indicate that the increase in the third light contribution turns out to be negligible (below 1\%) and the physical parameters of the stars in Tables 3 and 4 remain untained. And finally, the relative faintness of the $\mathrm{CU}$ Cnc's components when compared with other stars of similar mass is somewhat enhanced if the light from the close companion is subtracted. However, the magnitude differences in the $V$ and $K$ bands discussed in Sect. 7 increase by only $\approx 0.02$ $0.04 \mathrm{mag}$.

Acknowledgements. Thanks are due to M. I. Andersen \& R. J. Irgens who kindly prepared and performed CCD observations of CU Cnc with the Nordic Optical Telescope at La Palma. I am most grateful to E. F. Guinan, G. Torres, A. Giménez, C. Jordi, J. V. Clausen, D. Fernández, F. Arenou, and R. Estalella for their invaluable help and fruitful discussions during the course of this work. The photoelectric observations in this publication have been obtained with the Four College APT, which is supported by NSF grants AST95-28506 and AST-0071260. The ING support astronomers are thanked for carrying out the spectroscopic service observations used in this study. The William Herschel Telescope is operated on the island of La Palma by the Isaac Newton Group in the Spanish Observatorio del Roque de los Muchachos of the Instituto de Astrofisica de Canarias. This publication makes use of data products from the Two Micron All Sky Survey, which is a joint project of the University of Massachusetts and the Infrared Processing and Analysis Center/California Institute of Technology, funded by the National Aeronautics and Space Administration and the National Science Foundation. This research has made use of the SIMBAD database, operated at CDS, Strasbourg, France. This research has made use of NASA's Astrophysics Data System.

\section{References}

Allard, F., Hauschildt, P. H., Alexander, D. R., \& Starrfield, S. 1997, ARA\&A, 35, 137

Allard, F., Hauschildt, P. H., \& Schwenke, D. 2000, ApJ, 540, 1005

Andersen, J. 1991, A\&ARv, 3, 91

Anosova, J. P., \& Orlov, V. V. 1991, A\&A, 252, 123

Baraffe, I., Chabrier, G., Allard, F., \& Hauschildt, P. H. 1998, A\&A, 337,403

Barrado y Navascués, D. 1998, A\&A, 339, 831

Barrado y Navascués, D., Fernández-Figueroa, M. J., García López, R. J., de Castro, E., \& Cornide, M. 1997, A\&A, 326, 780

Barrado y Navascués, D., Stauffer, J. R., \& Patten, B. M. 1999, ApJ, 522, 53

Bergbusch, P. A., \& VandenBerg, D. A. 2001, ApJ, 556, 322

Bessell, M. S. 1979, PASP, 91, 589

Bessell, M. S., \& Brett, J. M. 1988, PASP, 100, 1134

Bessell, M. S., Castelli, F., \& Plez, B. 1998, A\&A, 333, 231

Bodenheimer, P. 1965, ApJ, 142, 451

Carpenter, J. M. 2001, AJ, 121, 2851

Cayrel de Strobel, G., Soubiran, C., \& Ralite, N. 2001, A\&A, 373, 159

Charbonnel, C., Däppen, W., Schaerer, D., et al. 1999, A\&AS, 135, 405

Claret, A. 2000a, A\&A, 359, 289

Claret, A. 2000b, A\&A, 363, 1081

Cousins, A. W. J. 1976, Mem. R. Astron. Soc., 81, 25

D'Antona, F., \& Mazzitelli, I. 1984, A\&A, 138, 431

D’Antona, F., \& Mazzitelli, I. 1997, Mem. Soc. Astron. Ital., 68, 807

Delfosse, X., Forveille, T., Mayor, M., Burnet, M., \& Perrier, C. 1999a, A\&A, 341, L63 (D99)
Delfosse, X., Forveille, T., Beuzit, J.-L., et al. 1999b, 344, 897

Delfosse, X., Forveille, T., Ségransan, D., et al. 2000, A\&A, 364, 217

Duncan, D. K. 1981, ApJ, 248, 651

Edvardsson, B., Andersen, J., Gustafsson, B., et al. 1993, A\&A, 275, 101

Elias, J. H., Frogel, J. A., Matthews, K., \& Neugebauer, G. 1982, AJ, 87,1029

Elias, J. H., Frogel, J. A., Hyland, A. R., \& Jones, T. J. 1983, AJ, 88, 1027

ESA 1997, The Hipparcos and Tycho Catalogues, ESA SP-1200

Fitzpatrick, E. L. 1999, PASP, 111, 63

Forveille, T., Beuzit, J.-L., Delfosse, X., et al. 1999, A\&A, 351, 619

Gagne, M., Caillault, J.-P., \& Stauffer, J. R. 1995, ApJ, 445, 280

Girardi, L., Bressan, A., Bertelli, G., \& Chiosi, C. 2000, A\&AS, 141, 371

Granzer, T., Schüssler, M., Caligari, P., \& Strassmeier, K. G. 2000, A\&A, 355, 1087

Greaves, J. S., Holland, W. S., Moriarty-Schieven, G., et al. 1998, ApJ, 506, L133

Güdel, M., Schmitt, J. H. M. M., Bookbinder, J. A., \& Fleming, T. A. 1993, ApJ, 415, 236

Hatzes, A. P. 1995, in Stellar Surface Structure, ed. K. G. Strassmeier (Vienna: Institut für Astronomie), IAU Symp., 176, 90

Hauschildt, P. H., Allard, F., \& Baron, E. 1999, ApJ, 512, 377

Holland, W. S., Greaves, J. S., Zuckerman, B., et al. 1998, Nature, 392, 788

Jeffries, R. D., \& Tolley, A. J. 1998, MNRAS, 300, 331

Klinglesmith, D. A., \& Sobieski, S. 1970, AJ, 75, 175

Leggett, S. K. 1992, ApJS, 82, 351

Leggett, S. K., Allard, F., Berriman, G., Dahn, C. C., \& Hauschildt, P. H. 1996, ApJS, 104, 117

Lejeune, T., Cuisinier, F., \& Buser, R. 1998, A\&AS, 130, 65

Maceroni, C., \& Rucinski, S. M. 1997, PASP, 109, 782

Martin, C., Mignard, F., Hartkopf, W. I., \& McAlister, H. A. 1998, A\&AS, 133, 149

Mazeh, T., Latham, D. W., Goldberg, E., et al. 2001, MNRAS, 325, 343

Metcalfe, T. S., Mathieu, R. D., Latham, D. W., \& Torres, G. 1996, ApJ, 456, 356

Milone, E. F., Stagg, C. R., \& Kurucz, R. L. 1992, ApJS, 79, 123

Palla, F., \& Stahler, S. W. 1999, ApJ, 525, 772

Pavlenko, Y. V., Rebolo, R., Martín, E. L., \& García López, R. J. 1995, A\&A, 303, 807

Ribas, I. 2003, A\&A, in press

Ribas, I., Giménez, A., Torra, J., Jordi, C., \& Oblak, E. 1998, A\&A, 330,600

Schmitt, J. H. M. M., Fleming, T. A., \& Giampapa, M. S. 1995, ApJ, 450,392

Ségransan, D., Delfosse, X., Forveille, T., et al. 2000, A\&A, 364, 665

Siess, L., Forestini, M., \& Dougados, C. 1997, A\&A, 324, 556

Soderblom, D. R., Oey, M. S., Johnson, D. R. H., \& Stone, R. P. S. 1990, AJ, 99, 595

Soderblom, D. R., Duncan, D. K., \& Johnson, D. R. H. 1991, ApJ, 375,722

Stauffer, J. R., Barrado y Navascués, D., Bouvier, J., et al. 1999, ApJ, 527,219

Swenson, F. J., Faulkner, J., Rogers, F. J., \& Iglesias, C. A. 1994, ApJ, 425, 286

Torres, G., \& Ribas, I. 2002, ApJ, 567, 1140 (TR02)

Voges, W., Aschenbach, B., Boller, T., et al. 1999, A\&A, 349, 389

Yi, S., Demarque, P., Kim, Y.-C., et al. 2001, ApJS, 136, 417

Young, A., Skumanich, A., Stauffer, J. R., Harlan, E., \& Bopp, B. W. 1989, ApJ, 344, 427

Weis, E. W. 1991, AJ, 101, 1882

Wilson, R. E., \& Devinney, E. J. 1971, ApJ, 166, 605 (W-D) 